\title{
A metastable phase of tin in 3D integrated circuit solder microbumps
}

\author{
Yingxia Liu ${ }^{1}$, Nobumichi Tamura ${ }^{2}$, Dong Wook $\mathrm{Kim}^{3}$, Sam $\mathrm{Gu}^{3}$, and K. N. Tu${ }^{1, \text { a) }}$ \\ ${ }^{I}$ Dept. of Materials science and Engineering, UCLA, Los Angeles, CA 90095-1595 \\ ${ }^{2}$ Advanced Light Source, Lawrence Berkeley National Lab., Berkeley, CA 94720 \\ ${ }^{3}$ Qualcomm, San Diego, CA 92121
}

\begin{abstract}
A metastable phase of $\mathrm{Sn}$ has been found to co-exist with $\beta$-Sn in Pb-free $\mathrm{SnAg}$ microbumps in 3D integrated circuit technology. Synchrotron microbeam x-ray diffraction, high-resolution TEM imaging and selected-area electron diffraction were used to confirm the metastable phase, which has a orthorhombic lattice, with lattice parameter $\mathrm{a}=0.635 \mathrm{~nm}, \mathrm{~b}=0.639$ $\mathrm{nm}$, and $\mathrm{c}=1.147 \mathrm{~nm}$. Its composition is Sn containing a few percent of Ni. A higher rate of nucleation might have enabled its formation.
\end{abstract}

\section{Key words}

Lead-free solder; metastable phases; electronic packaging; joining

In the era of big data and internet of things, mobile consumer electronic products are ubiquitous. To enable broader applications with wider bandwidth, more sensing, faster response, lower power consumption, and smaller form factor, the current chip technology is unable to meet these needs, because the trend of Moore's law in miniaturization is approaching

a) Electronic email: kntu@ucla.edu 
its limit. The most promising way to overcome the limit is to combine chip technology and packaging technology by stacking chips in 3D integrated circuit (IC). The challenging technical issue in 3D IC is the vertical interconnects between stacking chips. To do so, new structure components in packaging technology have been introduced. They are the throughSi-via (TSV) chips and the microbump solder joints [1-6]. With very intensive R\&D efforts, mass production of 3D IC is not yet on the horizon. This is because joule heating and reliability are of concern, and our knowledge of these new structure components is far from complete. In this letter, we report the finding of a new metastable phase of $\mathrm{Sn}$ in $\mathrm{Pb}$-free solder microbumps. To our understanding, the existence of this phase in solder joint is unknown.

Figure 1 shows a schematic diagram of the cross-section of two neighboring microbumps by using the thermal compression bonding method. To produce them, the top half and the bottom half of microbumps on Si are made by sequential depositions of $\mathrm{Cu}, \mathrm{Ni}$, and $\mathrm{SnAg}$ solder of $2 \%$ of $\mathrm{Ag}$. Then, underfill is dispensed before flipping the top half over the bottom half. With careful alignment, the bonding between the solder layers on the top half and on the bottom half occurs at a temperature slightly above the melting point of the $\mathrm{Pb}$-free solder [7]. During the bonding, a compression force about 100 Newton is applied on about 1000 microbumps of diameter of $20 \mu \mathrm{m}$. The compressive stress is about $300 \mathrm{MPa}$. It is applied to make sure that the upper and lower solder layers of all the microbumps in the array are in direct contact for bonding. This is because the TSV chip is thin, about $50 \mu \mathrm{m}$ in thickness, so it tends to buckle. Thus, the compression will reduce any gap between the top and bottom solder layers so that a uniform bonding can occur over all the microbumps. Although the process is using compression, the pressure is small. Actually, gap stoppers are used to define the gap between two stacking chips and they limit the compression. In cooling, the top die is rapidly cooled down at about $200{ }^{\circ} \mathrm{C} / \mathrm{sec}$ to increase thermal compression bonding throughput.

Since underfill is applied before bonding, some of the underfill is trapped in the solder joint. In addition, the compression will squeeze out the molten solder, so that a donut-type extrusion of solder will form a ring around the circumference of the microbump, as depicted in Fig. 1. Figure 2(a) shows a focused ion beam (FIB) cross-sectional image of a microbump, in which the filler trap in the middle and the extrusion at both ends of the solder joint are clear. In the FIB image, the microstructure of the $\mathrm{Cu}$, the $\mathrm{Ni}$, the intermetallic compound (IMC) $\mathrm{Ni}_{3} \mathrm{Sn}_{4}$, and the un-reacted solder can be seen. Figure 2(b) is SEM image of half of the cross-section of another microbump. No doubt, the filler trap and the extrusion are unique features of this type of solder joint. In the filler trap, some glass beads in the underfill can be seen. The thickness of the trap is about the diameter of a bead, which is about $100 \mathrm{~nm}$, and the width of the trap is about the diameter of the 
microbump. We note that there is no filler trap in the extrusion at both ends. Since the filler trap is a poor electric conductor, the microbump would have had high resistance if electric current had to go through the filler trap. However, the extrusion provides an alternative path for electrical conduction. Thus the solder extrusion is important.

When we examine the microstructure in the extrusion as well as in the unreacted solder around the filler trap, they seem to have a mixture, revealed by different indexing in electron back-scattering diffraction (EBSD). The EBSD images indicate that there are two different Sn phases; one is $\beta$-Sn but the other phase of Sn cannot be indexed as $\beta$-Sn. By using electron probe for compositional measurement as shown in Fig. 2(c) for $\beta$-Sn and Fig. 2(d) for the other phase, we confirm that both of them are nearly $100 \% \mathrm{Sn}$. However, we found that there are a few percent of Ni as well as some Au in the other phase of Sn. The presence of Au is puzzling; may be an issue in processing control of the microbumps. In Figure 2(a) and 2(b), we have labeled the $\beta$-Sn grains in the extrusion region. We note that in the SEM image in Figure 2(b), the $\beta$-Sn grain shows a very sharp contrast with the other Sn phase. This is because ion beam etches the $\beta$-Sn, resulting in a rough surface, so we can see a clear morphological difference between them in Fig, 2(b). The contrast in FIB image in Fig. 2(a) is because of the channeling effect.

In order to have a definitive identification of the crystal structure of the other Sn phase, we cut out tiny pieces of it and used synchrotron radiation micro beam x-ray diffraction at BL12.3.2 of the Advanced Light Source, Berkeley, to obtain clear Laue diffraction patterns. These pieces were scanned under a 1 micron size polychromatic beam with an energy band pass of 5 to $22 \mathrm{KeV}$ [8]. The indexing of the Laue patterns was performed using the XMAS software [9]. One of the indexed patterns is shown in Figure 3. The accuracy of indexation has been checked and the absolute lattice parameters were measured by performing monochromatic scans on the sample to measure the energy (wavelength) of each reflection. It is indexed as orthorhombic unit cell with lattice parameter of $a=0.635 \mathrm{~nm}, \mathrm{~b}=0.639 \mathrm{~nm}$, and $\mathrm{c}=1.147 \mathrm{~nm}$. The space group is Aba2. Table 1 lists the calculated value of interplanar spacing of the orthorhombic structure. The structure is probably isostructural with those of $\mathrm{Sn}_{4} \mathrm{Pt}, \mathrm{Sn}_{4} \mathrm{Au}$, and $\mathrm{Sn}_{4} \mathrm{Pd}$ with Ni replacing the noble metal $[10,11]$.

Knowing the structure of the metastable phase of Sn, we further confirm it by high resolution TEM images and selected area electron diffraction. Two pairs of image and its corresponding electron diffraction pattern are shown in Figure 4(a) and (b) as well as Fig, 4(c) and (d), respectively. The pattern in Fig. 4(b) has a near 4-fold symmetry, and its zone axis is [001] or the c-axis. In this pattern, we can measure the interplanar spacing values to determine the lattice parameter "a" (and "b") in the tetragonal structure, but not the lattice parameter of "c". The pattern in Fig. 4(d) has a near 2-fold symmetry, 
and we can determine "c". Its zone axis is $[0,-3,1]$. The lattice parameter values are in good agreement, within $5 \%$, with those determined from synchrotron radiation micro beam x-ray diffraction.

We recall that the equilibrium phases of pure $\mathrm{Sn}$ are known to be $\beta$-Sn (gray Sn or diamond $\mathrm{Sn}$ ) and $\beta$-Sn (white or metallic Sn). Besides, under a pressure of $110 \mathrm{kbar}$ at room temperature, $\beta$-Sn changes to a high pressure $\mathrm{Sn}$ phase, which has a body-centered-cubic crystal lattice. The lattice parameter is about $0.35 \mathrm{~nm}$. The phase change can also occur under pressure about $40 \mathrm{kbar}$ at $300{ }^{\circ} \mathrm{C}$, but the high pressure phase was found to have a body-centered-tetragonal crystal lattice with the ratio of $\mathrm{c} / \mathrm{a}=0.91$. It is worth noting that under high pressure, the melting point of $\beta$-Sn increases with pressure and at $30 \mathrm{kbar}$, and it is above $300{ }^{\circ} \mathrm{C}[12-14]$.

In addition, a $\gamma$-Sn phase (gamma Sn) is known, which forms upon rapid-quenching of an alloy of Sn having a few percent of other metals such as Ag. The cooling rate in the quenching is about $10^{6}{ }^{\circ} \mathrm{C} / \mathrm{sec}$. The $\beta$-Sn has a simple hexagonal unit cell of space group of $\mathrm{P} 6 / \mathrm{mmm}$, with lattice parameter of $\mathrm{a}=0.3212 \mathrm{~nm}$ and $\mathrm{c} / \mathrm{a}=0.9312[15,16]$.

The detection of the metastable phase of Sn in microbumps is very unusual because solder joints have been widely used in electronic packaging technology for a very long time, so we tend to assume that we know everything about solder alloys and solder joints. Nevertheless, the presence of the metastable phase presents a great reliability concern because it is not an equilibrium phase. When it transforms, the temperature as well as the molar volume change will affect the stressstrain state of the microbumps. The question of why can it form will require a detailed systematic study.

We recall that the thermal compression bonding of microbumps is different from the normal reflow process in solder joint formation, which has no applied compression and tends to cool down gradually. Still, we have no reason to expect the effect of high rate of rapid quenching and the effect of high pressure on formation of the metastable phase of Sn in microbumps. However, what is unique in the thermal compression bonding is the extrusion of molten solder which solidifies to form a donut-type ring around the circumference of the microbump. The donut ring has a large interface with the underfill, in turn a larger cooling rate when heat is being conducted away from the top die and underfill. If we assume that the metastable phase has a higher melting point than the $\beta$-Sn, relative speaking it has a much larger undercooling than $\beta$ Sn from the point of view of nucleation during the solidification of the microbump. We speculate that the metastable phase forms because of its higher rate of nucleation than that of $\beta$-Sn. It is very likely that the effect of Ni UBM on the nucleation should be considered too. Because the metastable phase has a structure which is probably isostructural to those of $\mathrm{Sn}_{4} \mathrm{Pt}$, 
$\mathrm{Sn}_{4} \mathrm{Au}$ and $\mathrm{Sn}_{4} \mathrm{Pd}$, the metastable phase could be an intermetallic compound of $\mathrm{Sn}_{4} \mathrm{Au}$ with Ni replacing the noble metal. While the metastable phase is the dominant phase in the remaining solder in the microbump, it co-exists with the minor phases of $\beta-\mathrm{Sn}$ and $\mathrm{Ag}_{3} \mathrm{Sn}$.

Typically, a metastable phase forms when the formation of the equilibrium phase ( $\beta$-Sn here) was suppressed due to kinetic reasons. For example, rapid quenching has been used to produce many amorphous alloys. What is of interest here is that we found the metastable phase co-exists with the equilibrium phase of $\beta$-Sn, as shown in Fig. 2. We do not know the sequence of their formation on the basis of the morphology of Fig. 2, whether the metastable phase or the $\beta$-Sn forms first. Nevertheless, it is reasonable to assume the metastable phase forms first. Otherwise, it has no thermodynamic reason to form after $\beta$-Sn. This metastable phase seems quite stable; it resists FIB etching better than $\beta$-Sn, and also it resists electromigration better. We will use differential scanning calorimetry to measure its stability. Also we will need to study its rate of reaction with $\mathrm{Ni}$ and $\mathrm{Cu}$ in solder joints. These studies will be reported elsewhere.

In summary, we found a metastable phase of $\mathrm{Sn}$ in microbumps formed by thermal compression bonding with $\mathrm{Ni}$ as the under-bump-metallization. It has orthorhombic lattice with $\mathrm{a}=0.635 \mathrm{~nm}, \mathrm{~b}=0.639 \mathrm{~nm}$, and $\mathrm{c}=1.147 \mathrm{~nm}$. We used synchrotron radiation micro beam x-ray diffraction to determine the structure, which has been confirmed by selected area electron diffraction. The composition of this phase has been inspected by electron micro probe to be almost $\mathrm{Sn}$, but it contains a few percent of Ni and some Au and Pd.

\section{Acknowledgement}

The authors at UCLA would like to acknowledge the financial support of project 77630 by Qualcomm. All of us acknowledge the technical support of Chi Ma at Caltech for EBSD study, Sergey Prikhodko at MSE, UCLA for SEM and TEM and Noah Bodzin at nanolab, UCLA for FIB study. The Advanced Light Source (ALS) is supported by the Director, Office of Science, Office of Basic Energy Sciences, of the U.S. Department of Energy under Contract No. DE-AC0205CH11231 at the Lawrence Berkeley National Laboratory (LBNL). 


\section{References}

1. S. Q. Gu, P. Marchal, M. Facchini, in Proceedings of Electron Devices Meeting, (2008), pp. 1-4.

2. D. W. Kim, R. Vidhya, B. Henderson, in Proceedings of Electronic Components and Technology Conference, (2013), pp. $77-80$

3. M. J. Wolf, T. Dretschkow, B. Wunderle, in Proceedings of Electronic Components and Technology Conference, (2008), pp. 563-570.

4. Y. Liu, M. Li, in Proceedings of Electronic Components and Technology Conference, (2014), pp. 609-612.

5. R. Labie, P. Limaye, K. W. Lee, in Proceedings of Electronic System-Integration Technology Conference, (2010), pp. 15 .

6. K. N. Chiang, C. C. Lee, Appl. Phys. Lett. 88 (2006) 072102.

7. R. Agarwal, W. Zhang, P. Limaye, in Proceedings of Electronic Components and Technology Conference, (2010), pp. $858-863$

8. M. Kunz, N. Tamura, K. Chen, Rev. Sci. Instrum, 80 (2009) 035108.

9. N. Tamura, in: R. Barabash and G. Ice (Eds.), Strain and Dislocation Gradients from Diffraction, Imperial College Press, London, 2014, pp. 125

10. B. Kunnen, D. Niepmann, W. Jeitschko, J. of Alloys and Compounds, 309 (2000) 1

11. C. E. Ho, S. W. Lin, and Y. C. Lin, J. of Alloys and Compounds, 509 (2011) 7749.

12. A. Jayaraman, W. Klement, Jr., and G. C. Kennedy, Phys. Rev. 130 (1963) 540.

13. M. J. P. Musgrave, J. Phys. Chem. Solids, 24 (1963) 557.

14. J. D. Barnett, V. E. Bean, and H. T. Hall, J. Appl. Phys. 37 (1966) 875.

15. G. V. Raynor and J. A. Lee, Acta Met. 2 (1954) 616.

16. R. H. Kane, B. C. Giessen, and N. J. Grant, Acta Met. 14 (1966) 605. 


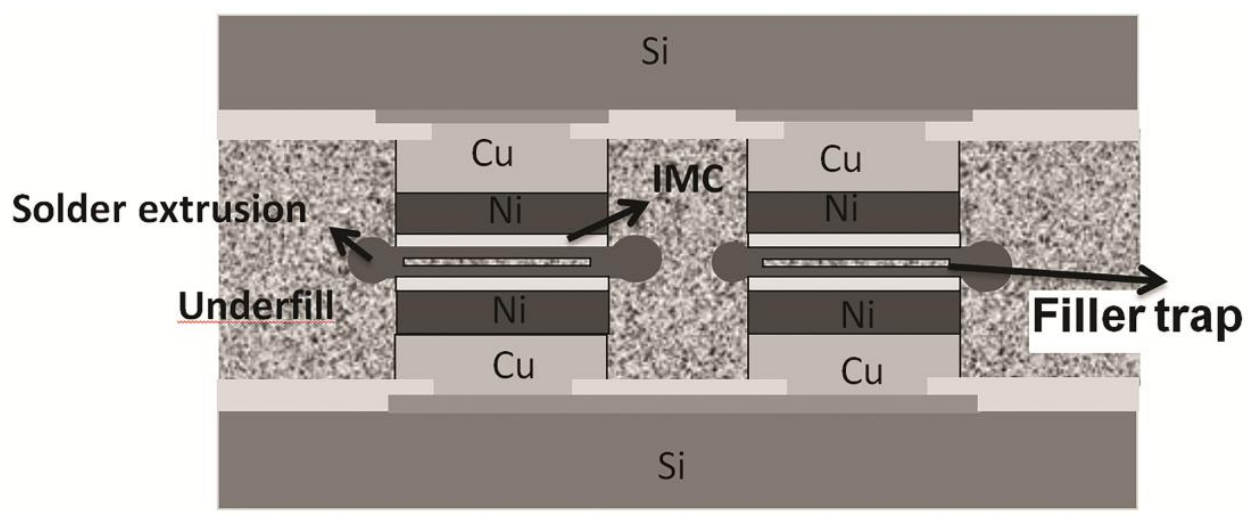

FIG. 1 Schematic diagram of thermal compression bonding showing the formation of filler trap and solder extrusion.
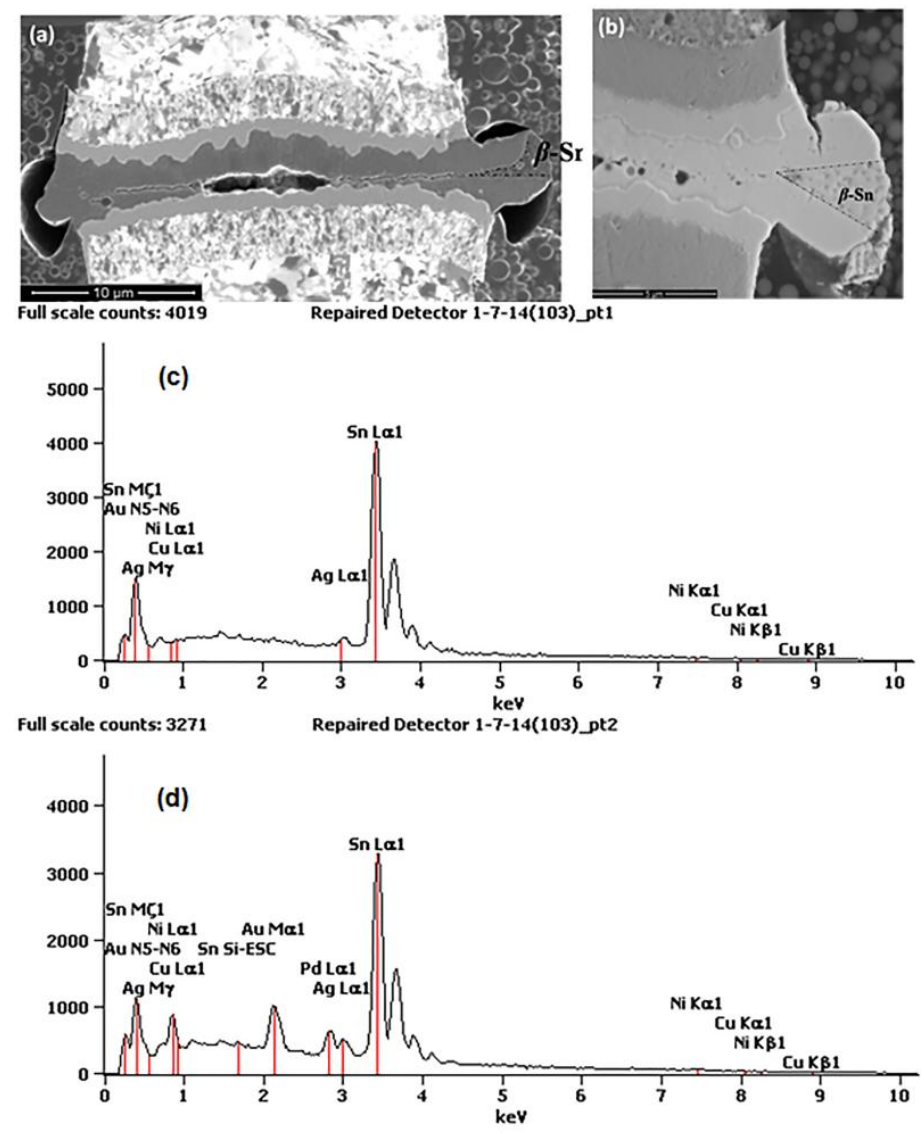

Fig. 2 (a) FIB image, (b) SEM image, and (c) and (d) EDX analysis of Sn in cross-section of a microbump. 


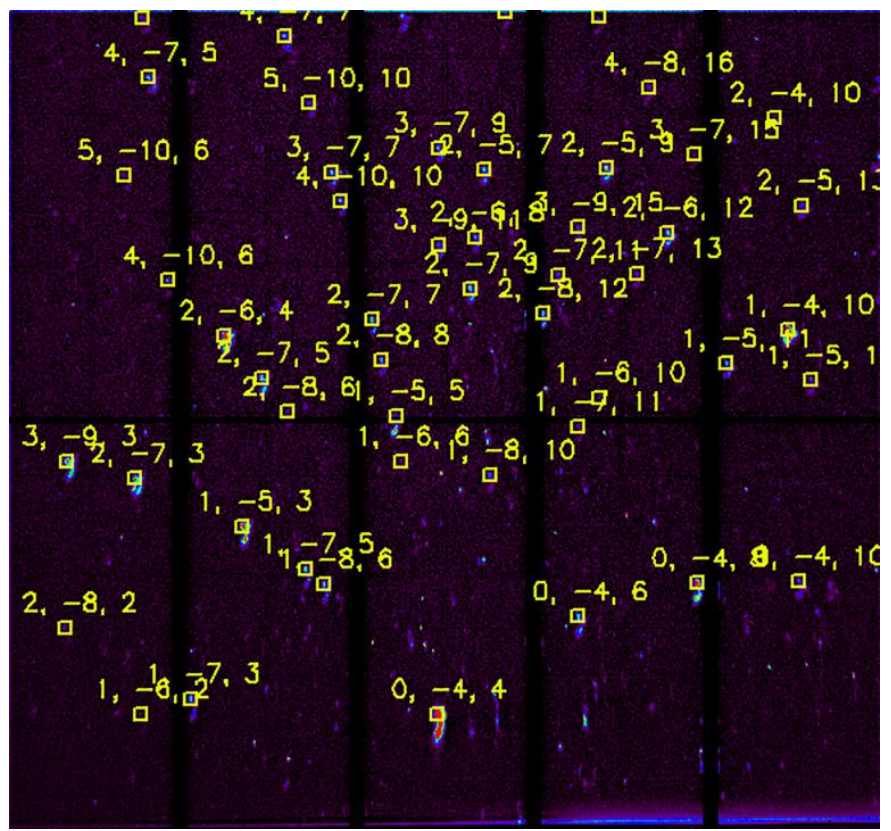

FIG. 3 Synchrotron radiation micro beam x-ray diffraction of metastable Sn phase indexes as orthorhombic unit cell with lattice parameter of $\mathrm{a}=0.635 \mathrm{~nm}, \mathrm{~b}=0.639 \mathrm{~nm}, \quad$ and $\mathrm{c}=1.147 \mathrm{~nm}$
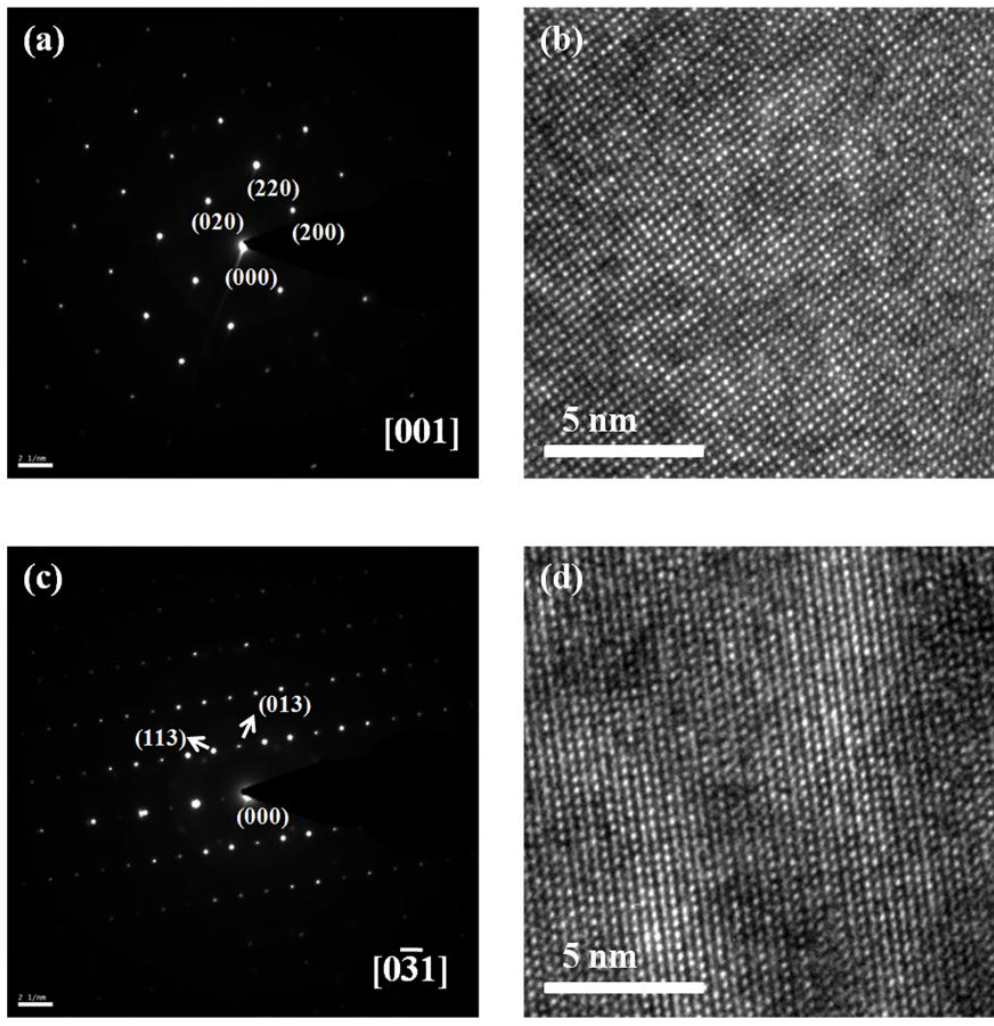

FIG. 4 Two pairs of lattice image and its corresponding electron diffraction pattern 
TABLE I. hkl reflections, interplanar spacing, and relative intensity of metastable Sn

\begin{tabular}{ccc}
\hline \hline (hkl) & d-spacing $(\AA)$ & Relative intensity \\
\hline 002 & 5.73270 & 100.0000 \\
111 & 4.19201 & 14.61959 \\
020 & 3.19570 & 4.62170 \\
200 & 3.17380 & 5.37955 \\
113 & 2.91404 & 5.89791 \\
004 & 2.86635 & 18.72840 \\
120 & 2.85437 & 22.15377 \\
022 & 2.79130 & 6.50030 \\
202 & 2.77667 & 2.59351 \\
213 & 2.28087 & 2.37076 \\
220 & 2.09600 & 0.79254 \\
\hline \hline
\end{tabular}

